\title{
Uncovering Financial Shenanigans: Benford's Law as a Computer Assisted Analytical Procedure
}

\author{
Nirosh Kuruppu ${ }^{1}$ \\ ${ }^{1}$ Asst. Professor, Department of Accounting, Sultan Qaboos University, Muscat, Oman \\ Correspondence: Nirosh Kuruppu, Department of Accounting, Sultan Qaboos University, PO Box 20, PC 123, \\ Muscat, Oman. E-mail: niroshk@squ.edu.om
}

Received: April 15, 2020

doi:10.5539/ijbm.v15n7p37

\begin{abstract}
PricewaterhouseCoopers (2020) has reported the highest level of economic crime in their comprehensive annual survey of the issue since it launched more than twenty years ago. Two thirds of respondents indicated that the costs of fraud can reach up to a million dollars each, amounting to approximately ten percent of their annual turnover. Inside perpetrators such as employees commit about 37 percent of these frauds. In this context, a technique known as Benford's Law can be cost-effectively applied to detect financial fraud which can be invaluable to auditors and other financial professionals. Benford's technique is founded on the mathematical distribution of integers found in nature and has been shown to be particularly efficient and cost-effective in financial fraud detection. The technique can swiftly flag suspicious transactions from lists of numbers that comprise millions of records when employed as a computer assisted auditing procedure. Despite this, Benford's Law is not widely used in accounting and finance. One of the key reasons for its limited use is because fraud investigators are often incognizant and unfamiliar with the method, and how it can be implemented in a fraud detection workflow. This paper set forth a concise and organised approach for implementing Benford's technique as an analytical procedure through the well-known IDEA generalised audit software to flag suspicious transactions, which can then be further investigated. Both the application of the method and its interpretation in situations of both compliance and non-compliance is discussed. The methodology proposed in this paper can be an indispensable aid for fraud investigators in view of the considerable costs associated with economic crime.
\end{abstract}

Keywords: economic crime, fraud detection, benford's law, analytical procedure, forensic accounting, audit software

\section{Introduction}

PricewaterhouseCoopers (PwC) in their latest Global Economic Crime Survey 2020 reports that 51\% of identified frauds exceeded US\$100,000, and the costs from fraud over the past 24 months exceeded US\$42 billion (PricewaterhouseCoopers, 2020). Crow Clark and Whitehall, an accountancy firm based in the UK also reports the high cost of fraud, stating that about ten percent of corporate revenues are wiped out by economic crime (Gee, 2018). In a similar vein, Lavion (2018) report a survey in the US where sixty four percent of participants indicated that their most significant fraud incident could see damages in the vicinity of US\$1 million.

The global impact of fraud is colossal. This is reported as being close to $\$ 3$ trillion dollars (Sweet, 2018). In this environment, a notable yet novel advancement is the identification of the usefulness of the Benford technique in detecting fraud. Benford's Law is established on the mathematically proven distribution of naturally existing integers, such as those found in accounting and finance. Prior studies have acknowledged its usefulness as a method that can assist investigators uncover fraud in accounting numbers (Kuruppu, 2019).

While most frauds (55\%) are committed by external parties, a significant proportion (37\%) are committed by internal perpetrators (PricewaterhouseCoopers, 2020). This represents a 5\% year on year increase over PwC's earlier fraud survey. In this context, external auditors and other investigators can immensely benefit from techniques that can assist in efficiently identifying situations where fraud may have occurred. Corporate management and internal auditors will benefit by having an efficient technique that can quickly identify potential internal occurrences of fraud, which can be the starting point for further detailed investigation (Kuruppu, 2019).

A common method employed by accounting executives to misreport financial statements is by concocting bogus 
journal entries. For instance, this might be by creating and recording non-existent purchases of inventory. Similarly, false expense claims may be used to hide the misappropriation of assets (Amouzegar \& Moshirvaziri, 2018; Pomykacz et al., 2017). When fraudsters succeed in merging fabricated entries among real journal entries, investigators face an uphill task (Nigrini, 2019; Simkin, 2010). This is a real issue for fraud investigators as the fraudulent entries may be hidden amongst millions of legitimate transactions, which makes accounting frauds very difficult to detect (Kuruppu, 2019; PricewaterhouseCoopers, 2020).

What is promising in this context is the fact that Benford's Law can "see through" these spurious accounting entries with little effort from the fraud investigator. This enables suspicious entries to be quickly identified and looked into more thoroughly (Collins, 2017; Singleton, 2011). Because the rates of naturally occurring integers differ from unnaturally formulated distributions such as when accounts are manipulated to commit fraud, the Benford technique can flag the latter as anomalies. For instance, an artificial distribution arises when a fraudster attempts to understate a series of expenses so that they do not exceed a particular threshold value (Kuruppu, 2019; Nigrini, 2019). These types of artificial distributions are effortlessly identified by the Benford technique.

A multitude of number distributions in accounting and finance comply with Benford's Law. Examples of these are number distributions from sales transactions, depreciation expenses and accounts receivables data (Kuruppu, 2019). The latter examples can be considered to be naturally occurring as long as the numerals are not restricted to particular values (Pomykacz et al., 2017). These types of number distributions are ideal candidates in fraud detection using the Benford method (Kruger \& Yadavalli, 2017; Kuruppu, 2019).

One of the main benefits of the Benford method is its efficacy and cost effectiveness. The method can be effortlessly implemented by a fraud examiner through a spreadsheet program or through generalised audit software (Kuruppu, 2019; Kyd, 2017; Pomykacz et al., 2017). This allows an investigator to determine if the numbers under examination needs to be looked at more thoroughly (Nigrini, 2018). Courts of law have also recognised Benford's analysis as admissible evidence (Pomykacz et al., 2017; Nigrini, 2019).

Several studies have shown the effectiveness of Benford's Law. For instance, both Collins (2017) and Kuruppu (2019) demonstrate in detail how the technique can be applied through Microsoft Excel to carry out the requisite analysis to detect suspicious transactions. Although the method can be implemented through a spreadsheet program, it is tedious and more prone to error due the number of intermediate steps involved. Using a spreadsheet for carrying out Benford's analysis requires specialist skill, which to a great extent can be eliminated by using one of the commonly available Generalised Audit Software.

Many audit software packages recognise the Benford method for uncovering accounting anomalies by integrating modules into the software. Two of the popular audit software on the market is IDEA ${ }^{\mathrm{TM}}$ and ACL ${ }^{\mathrm{TM}}$. Both these packages are able to report if transactions comply with Benford's distribution and isolate any anomalies for further investigation (Oregon Audit, 2016; Pomykacz et al., 2017). The main issue, however, is that many accounting professionals are unaware of the Benford method. Many investigators who are acquainted with the method lack a clear understanding of how to operationalize the technique to swiftly detect anomalies in accounting numbers that can be a starting point for more thorough investigation (Kuruppu, 2019).

This paper introduces a succinct approach that will permit accounting professionals to implement Bedford's Law as a computer assisted auditing procedure to flag suspicious amounts. A cost effective and swift method that can detect accounting anomalies can be of significant benefit to professional accountants, auditors and fraud examiners.

The paper is structured in the following manner. The next section examines Benford's Law, with a discussion on its usefulness for uncovering fraud. The relevance of the method as a computer assisted audit technique is also discussed. Section three presents a succinct approach for applying Benford's technique using two real world data sets. Both the compliance to Benford's Law indicating no anomalies is examined with instances of noncompliance indicating the presence of suspect amounts that need further investigation. Section four concludes the paper with implications for uncovering fraud and presents additional research opportunities.

\section{Background to Benford's Law}

The origins of Benford's Law date back to 1881, when Simon Newcomb, a Professor of Mathematics in the U.S. Navy observed the first pages of logarithm books were far more worn than later pages (Bhattacharya et al., 2011; Singleton, 2011). Lower level numbers occur in the first pages of logarithm books used at the time to aid in computations. This led Newcomb to postulate that earlier integers were used more than later numerals in the natural world, and he developed an ingenuous yet simple equation to summarise this fact. (Newcomb, 1881; Nigrini, 2019). This equation was:

$$
P\left(D_{1}=d\right)=\log _{10}\left(1+\frac{1}{d}\right)
$$


where $\mathrm{d}=$ natural integers inclusive of 1 to 9 , and $D_{1}$ is the ratio of integers beginning with the number $\mathrm{d}$.

This equation successfully summarised the occurrence of naturally occurring integers beginning with the digits 1 to 9 . However, Newcomb's observations were left largely neglected for a considerable period. This was because he did not have a proper explanation for his observations, in spite of having disseminated his findings in the American Journal of Mathematics (Hill, 1998; Moolman, 2017).

Newcomb's observations were later rediscovered by Frank Benford in 1938, a physicist in the United States (Singleton, 2011). He noticed the same phenomena in logarithm books (Benford, 1938). Benford theorised that earlier pages were more worn because lower level numbers occurred in the world with greater frequency. Therefore, earlier pages in logarithm books were looked up more (Benford, 1938; Nigrini, 2019).

To test his theory, Benford examined naturally occurring data from many different sources. Some 20,229 observations from geographic, scientific and demographic sources were examined (Benford, 1938; Silva et al., 2017). Benford's findings were fascinating, with the key observation that the leading digit of the examined numbers spread logarithmically and not arithmetically (Silva et al., 2017). The leading digit is the first digit in each number. For example, Australia has a population of 25,236,900 million. Therefore, the leading digit of Australia's population is 2. According to Benford, lower digits such as the number 1 appear more frequently in nature compared to higher digits such as the number 9. Larger digits such the number 9 occur with less frequency compared to earlier numerals (Kruger \& Yadavalli, 2017). Benford's leading digit observations are shown in Figure 1. The earlier digits such as the number 1 and 2 occur $30.1 \%$ and $17.6 \%$ of the time respectively in naturally occurring numbers. In contrast, numbers starting with the digit 9 only occurs about $4.6 \%$ of the time.

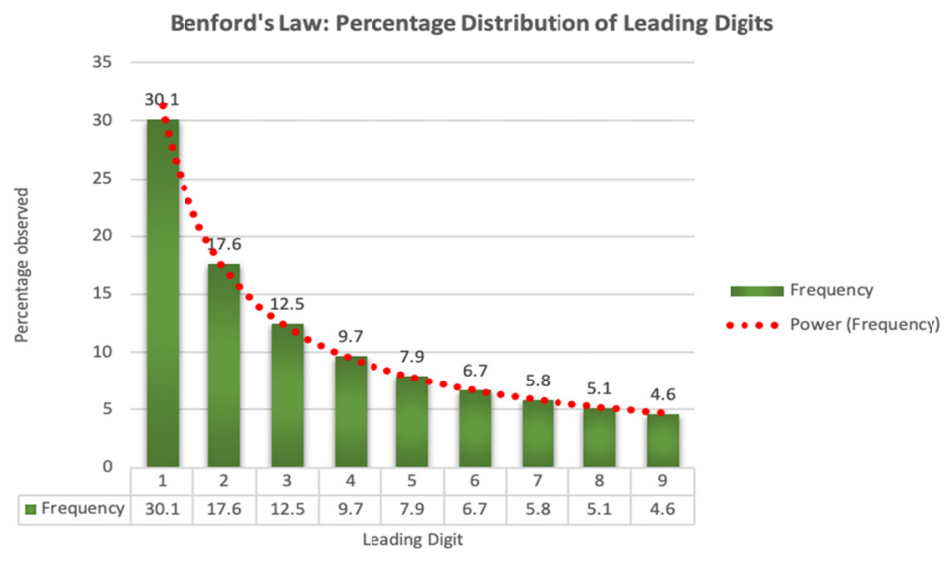

Figure 1. Occurrence of first digits in naturally occurring numbers

Despite the fact that Benford's Law has been around for a considerable period of time, its usefulness and possible applicability in accounting and finance was only recognised much later. Boyle (1994) showed that number lists subject to mathematical operators also obey the above described number proportions. Accounting numbers are frequently subject to mathematical operations. For instance, the value of total purchases is found by taking the product of the unit cost and quantity. A list of purchase transactions will therefore be expected to abide by Benford's distribution (Boyle, 1994; Durtschi et al., 2004). Conversely, non-compliance with Benford's distribution is an indication that there is a possible misstatement in the purchase transactions (Kuruppu, 2019).

Hill (1995) established that leading digits following Benford's Law are permutations of other distributions. If distributions are picked at random, and then samples are taken from these distributions, the first digit incidences of the collective samples will abide by Benford's expectation (Hill, 1998). This is expected even though the leading digits from individual samples may not comply with Benford's distribution (Durtschi et al., 2004; Hill, 1998). Hill (1995) offered a theoretical foundation for Benford's Law and presented its validity on both stock market and other accounting numbers.

Other studies have specifically shown the utility of Benford's Law in accounting. In $\mathbb{N e w}$ Zealand, Carslaw (1988) showed that published revenue numbers of listed companies in New Zealand did not comply with Benford's Law, indicating possible manipulation. Similar observations were reported for U.S. companies (Thomas, 1989). 
The potential of Benford's Law in identifying tax fraud was examined by Cho \& Gaines (2007), Watrin et al., (2008) and Nigrini $(1999,2019)$. The overarching conclusion from these studies was that Benford's Law can be used to identify number patterns indicative of manipulation in payable tax amounts. This can be the foundation for more detailed examination.

From an auditing perspective, Nigrini and Mittermaier (1997) developed six numerical tests based on Benford' Law that were subsequently used by Ernst \& Young to discover irregularities in different audit areas (IDEA, 2018). This study was instrumental in applying Benford's distribution in the form of a reasonableness check. Later, Weinberg and Busta (1998) demonstrated the method's efficacy as an audit tool. More recently, Carreira \& Silva (2016) demonstrated the real-world applicability of the method when used as part of a continuous audit program.

While the potential usefulness of Benford's Law to identify accounting anomalies has been recognised, it is only lately that its applicability to fraud detection has been thoroughly considered (Wells, 2013; Moolman, 2017). The assertion made by Chou $(2018$, p.2) that "Benford's law is widely applicable but not widely known..." is an apt depiction of the scope of its usage among accounting professionals. Nevertheless, it is promising that the technique's applicability is being increasingly recognised in accounting and finance (Kuruppu, 2019; Nigrini, 2019; Wells, 2013)

With the reported high levels of economic crime and fraud, a reliable and systematic implementation of Benford's technique has the potential to be immensely beneficial to accounting professionals such as auditors and fraud examiners (IDEA, 2018; Kruger \& Yadavalli, 2017; Kuruppu, 2019). ACL ${ }^{\mathrm{TM}}$ and IDEA ${ }^{\circledR}$, which are two of the noted generalised audit software on the market both implement Benford's Law. This can allow auditors and fraud examiners to examine millions of number records almost instantaneously for non-conformity with Benford's distribution indicating specific transactions that require more detailed examination.

With audit firms facing considerable pressure to reduce costs and increase efficiency, audit techniques based on information technology can significantly expedite audit efforts. Despite the increasing interest in such technologies, not many auditors are conversant with Benford's Law in the form of a computer based analytical procedure (Baker, 2009; IIARF, 2009; Kuruppu, 2019). Others recognise the usefulness of generalised audit software, but perceive features such as Benford's Law as difficult to use (Kim et al., 2009). The latter is a misconception as the method can be deployed in minutes to flag questionable transactions. Auditors' unfamiliarity with the technique means that they are not using an effective tool in the fraud fighting toolkit to its full potential. The current Global Economic Crime and Fraud Survey refer to various fraud fighting technologies as part of a rounded corporate anti-fraud programme (PricewaterhouseCoopers, 2020).

The objective of this paper is to present a systematic methodology to allow for a more pervasive use of Benford's Law as a computer assisted analytical procedure through IDEA ${ }^{\circledR}$. The methodology delineated here can be swiftly implemented to identify accounting anomalies that may warrant more detailed examination to eliminate the possibility of fraud. This can be invaluable to fraud investigators in light of the substantial increase in economic crimes in recent times. The following section presents this methodology.

\section{Applying Benford's Law in IDEA®}

Benford's Law holds for numbers occurring naturally such as in corporate sales or accounts receivables, if the amounts are not restricted by set boundaries. Amounts within a given number distribution that do not meet Benford's distribution such as when they are artificially created or manipulated will be flagged as anomalies. As such, it is important to utilise real world data to examine the efficacy of Benford's Law as an analytical technique.

IBM Watson Analytics provides several real-world corporate datasets that are ideal for this purpose and are in the public domain. Two of these datasets were utilised in this paper to show how Benford's Law can be applied through IDEA ${ }^{\circledR}$ to detect accounting anomalies. Moreover, the availability of the data on the IBM Watson website is helpful for reproducing the presented methodology. For clarity and conciseness, the methodology is summarised in three main steps as follows:

1. Project creation

2. Applying Benford's Law

3. Results interpretation

The methodology presented here will enable investigators to apply the Benford's technique consistently and with minimal steps to identify if the number distribution under examination contain anomalous amounts that warrant more scrutiny. The methodology presented here is from the logical perspective of an accounting 
professional whose objective is to determine if the reported revenues and receivable amounts are legitimate, and not artificially manipulated to commit fraud.

Both datasets described above on revenues and accounts receivables were in CSV format, with the first row containing the field name headers. IDEA always imports a copy of the original data into the workspace. This ensures that the integrity of the original data is maintained, as it cannot be subsequently changed or modified by IDEA. Benford's Law is administered on the two datasets and interpreted as follows.

\section{Step 1: Project creation}

The precursor to performing any analysis in IDEA is to import the required data into the IDEA workspace in the form of a Project. Once this is done, the Benford's Law analysis through IDEA is straightforward. It is therefore imperative that this first step is correctly carried out since the subsequent analysis depends on having the complete population of interest in IDEA. A project can be visualised as a virtual folder, where all the required data for the analysis is stored together with the results of the analysis.

(a) To create the project, start by clicking on the Create button on the Hometab, as shown below in Figure 2. Then enter a suitable name for the project under Project name and click on OK.

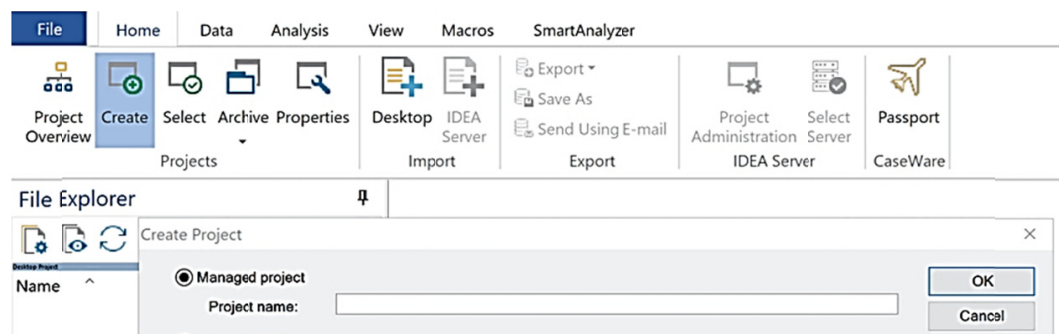

Figure 2. Creating an IDEA project

(b) The next step is to populate the Project with the necessary data for the Benford Law analysis. This entails importing the IBM sales and accounts receivables data into the project. Click on the Desktop button on the Hometab, which will open the Import Assistant that will guide the user to import the required files. Several file formats are supported, including source files in Excel, Access, PDF and Text formats. The CSV file used in this paper is classified in IDEA as a Text format. Upon selecting the correct format, specify the source file location to start the importation process as shown in Figure 3 below. The Import Assistant will present a preview of the data to be imported. It will also preselect the data type such as Delimited or Fixed length. If the data type is not correct, it is necessary to correct this before clicking on Next in the Import Assistant.

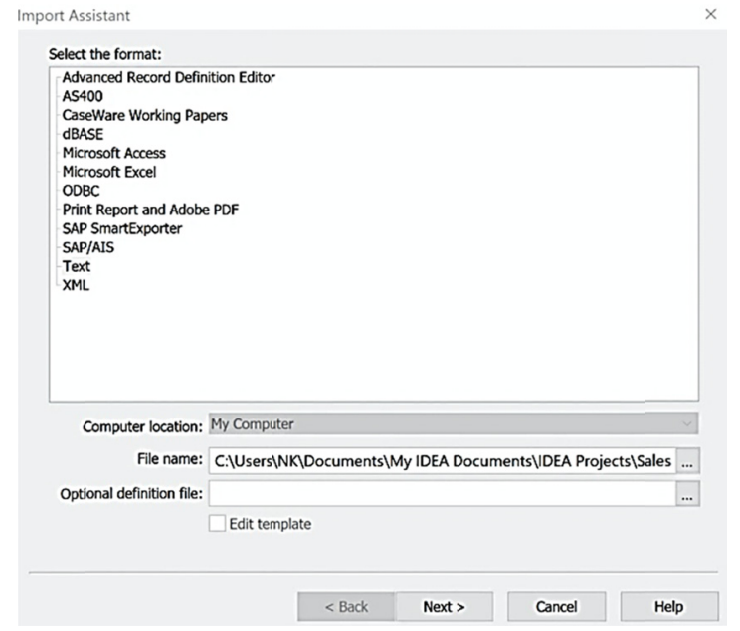

Figure 3. Specifying source data type and data location

(c) The next screen requires the user to check whether the data is correctly organised before it is copied into IDEA. This is shown once again in a preview screen, with the field separators shown as Comma, Colon, Tab and so on. IDEA is generally able to identify the correct field separators, and user input is only required if the default 
selection is not appropriate. If the data contain the field names in the first row, the user must be careful to select the checkbox specifying this, as shown below in Figure 4.

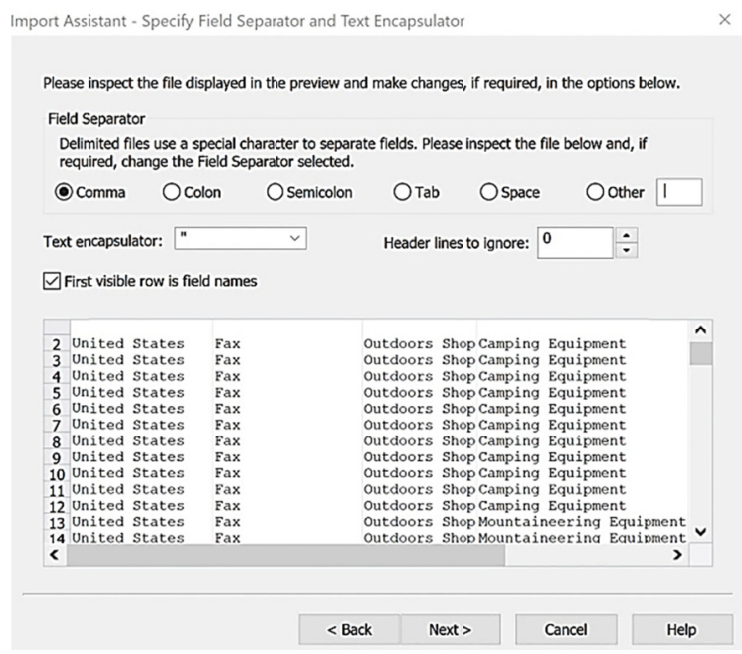

Figure 4. Specifying column separators and field names

(d) Before IDEA finally copies the data, the penultimate preview screen requires the user to check the data column types. These include specifying data types such as Character, Numeric and Date for each of the columns in the database. It is important to specify the correct types as some columns, such as those containing ID numbers should not be used in calculations by IDEA even though they comprise of numbers. Data columns that are not required for analysis can be excluded from importation by checking the "Do not import this field" checkbox. This will minimise errors in the analysis by only accessing the data needed. Clicking Next imports the data into the created project, and it will be displayed within IDEA as shown in Figure 5.

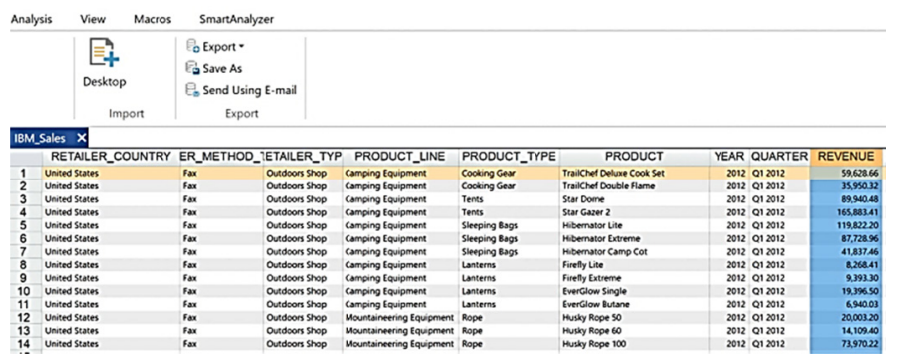

Figure 5. Imported data

Identical steps were used to also import the IBM accounts receivables data and run the Benford's tests. The final project file contained sales data with 5,000 instances, while the accounts receivables data comprised of 2,466 instances. These far exceeded the minimum number of 100 instances that is recommended to properly implement and interpret Benford's Law (Simkin, 2010).

\section{Step 2: Applying Benford's Law}

The procedure is run from the Analysis tab. Clicking on the Benford's Law icon brings up the options window, as shown in Figure 6. Several selections need to be made here. 


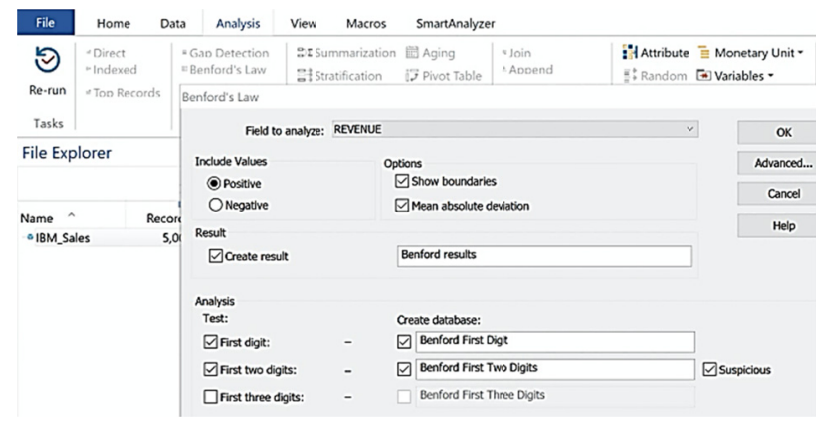

Figure 6. Specifying the Benford tests

(a) In the 'Field to analyse' dropdown list, select the column of data on which to conduct the Benford's Law analysis. Here, Revenue has been selected. The other options should be left to their default values.

(b) Select the 'Create result' checkbox and provide a name for the results. This will ensure that the results of the analysis will be appended to the project for later review.

(c) In the Analysis section, enable the 'First digit' test and the 'First two digits' test. Give a name for the ensuing analysis that will be added to the project. The first digit and first two digits tests are known as primary tests. These are the most practical due to their efficiency. It is also necessary to select the checkbox next to 'Suspicious'. This will result in IDEA flagging and identifying anomalies in the number distribution that need to be further investigated. Clicking on OK will run both tests, and by default, IDEA will display the results of the First digit test graphically.

\section{Step 3: Results interpretation}

Interpreting Benford's analysis in IDEA starts with the graphical summaries. These are analysed as follows, starting with the sales data results and then the accounts receivables data.

(a) The First digit test for the sales data is shown below in Figure 7. The bars on the chart shows the frequency of occurrence for each digit from the number 1 through to number 9. The horizontal axis shows the first digits of the transactions, while the vertical axis summarises the count of those first digits. Overlaid on this chart in red is Benford's expected count of numbers for the distribution, including the minimum and maximum boundaries. The first digit results show that the distribution follows Benford's expected rates. The general conclusion, which in this case is 'Acceptable conformity,' is shown on the upper left-hand corner of the chart. Therefore, the First digit test on the revenue numbers do not flag any anomalies that justify further investigation.

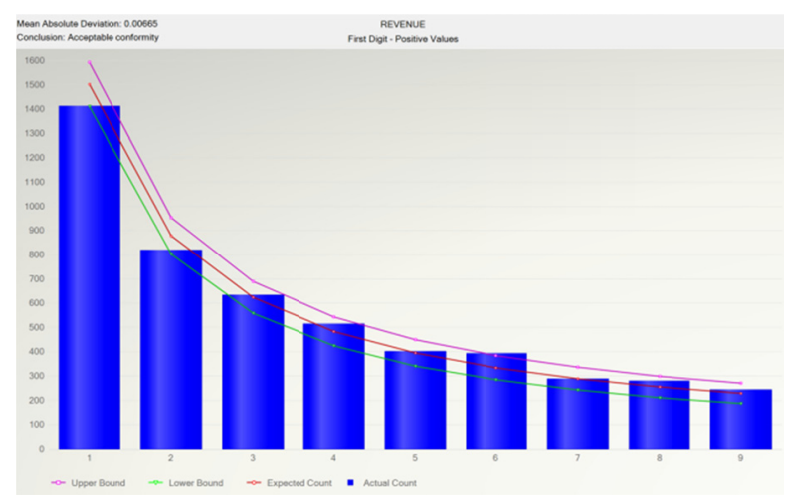

Figure 7. Acceptable conformity in Benford's First digit test

(b) Although the First digit test above does not flag any anomalies, it is recommended to additionally examine the First two digits test as well. This can indicate anomalies that might not appear in the initial test, which is a high-level assessment. Clicking on the two-digit test graphically shows the result of this assessment. This is shown in Figure 8. 


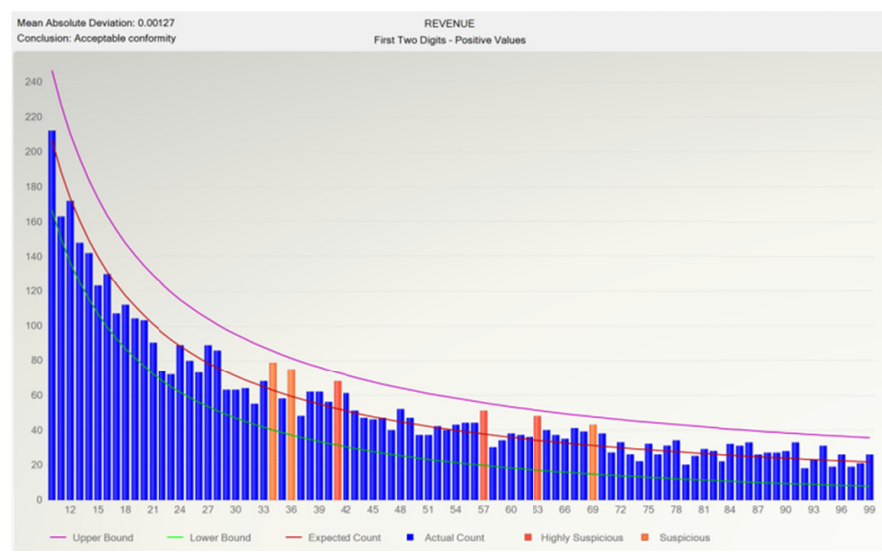

Figure 8. Acceptable conformity in Benford's First two digits test

It immediately becomes clear that several combinations of numbers are flagged for further investigation, as either 'Suspicious' or 'Highly Suspicious'. For instance, 79 numbers starting with the first digits 3 and 4 consecutively have been flagged as suspicious. This is from a total of 5,000 instances in the examined data. The investigator can right-click on any of the suspicious bars on the chart to get an option to extract the questionable records for more detailed identification and follow up, as shown below in Figure 9. These instances can be saved into the project for documentation and later review. The flagged numbers in the Sales data are not an automatic indicator that a fraud has occurred. In this illustration, the large frequency of numbers starting with 34 might have a perfectly logically explanation, such as most of the product sales start with 34 and its multiples. However, this must be verified as such. As shown in Figure 9, despite the flags, the number distribution still complies with Benford's Law as the displayed conclusion is "Acceptable conformity". The investigator needs to exercise professional judgement as to whether further action and scrutiny of the identified numbers are needed.

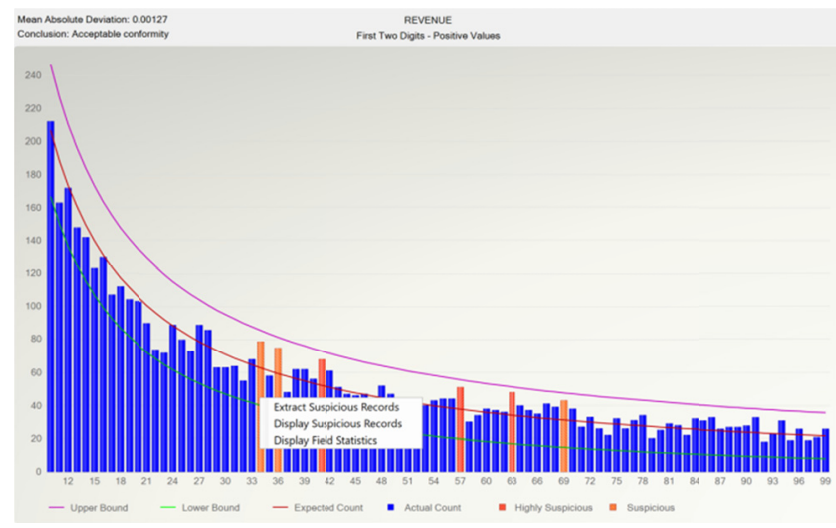

Figure 9. Extracting suspicious records

A clear case of non-conformity with Benford's Law emerges when the above steps are also run on the IBM accounts receivables data. The analysis starts by examining the First digit test, the summary of which is shown in Figure 10. The actual count of the receivables data does not adhere to the expected distribution, thus alerting the investigator that these numbers need to be more thoroughly scrutinised. The graphic clearly marks the distribution as non-confirming in the upper left-hand corner. 


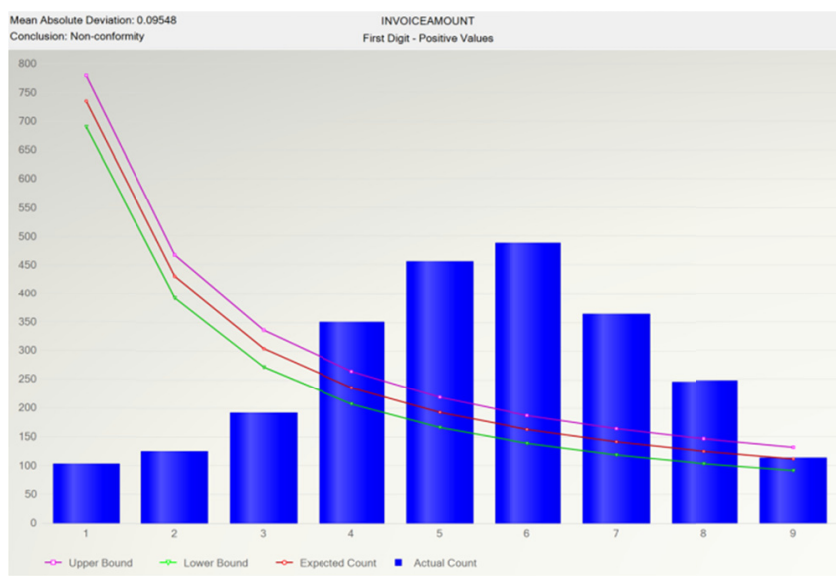

Figure 10. Non-conformity in Benford's First digit test

Since the First digit assessment indicate anomalies, it is essential that the First two digits test is examined to specifically identify where the anomalies are present and most prevalent. The result of this test is shown in Figure 11. The First two digits test notifies the investigator of non-compliance. The test identifies that accounts receivable transactions starting with the numerals 56, 60,61, 64, 68 and 72 need to be scrutinized in more detail, mindful that these numbers might be misstated, either deliberately or by error. Right-clicking on the suspect bars enables the extraction of the anomalous transactions for more detailed investigation.

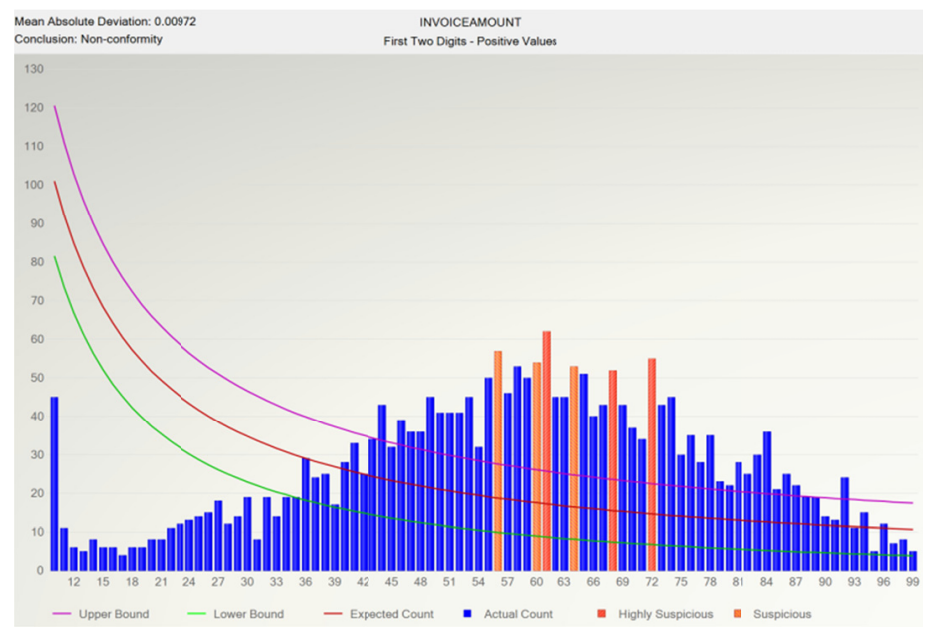

Figure 11. Non-conformity in Benford's First two digits test

It is apparent from the above analysis that an auditor can effectively apply Benford's Law in the form of an initial reasonableness check to obtain a high-level understanding of areas that needs to be focussed on during the audit. The auditor will not be overly concerned with the sales figures, as they comply with Benford's Law. However, the auditor will recognise that more audit effort will be required in the receivables area, as there is a greater chance that these numbers might contain irregularities. This will allow the auditor to reassess the risk of material misstatement for the client and ensure that the collected evidence supports the reached conclusions.

\section{Summary and Implications}

Incidences of economic crime and fraud have significantly increased over the past two decades, with PwC putting the number at USD \$42 billion in their latest fraud survey (PricewaterhouseCoopers, 2020). In this context, Benford's Law was advanced as an analytical technique that can uncover irregularities in corporate accounting numbers. Instead of sampling transactions, this method can be rapidly implemented on entire populations of interest through information technology. Moreover, analysis from Benford's Law is accepted as evidence in courts of law and it also implemented in well-known generalised audit software such as ACL and IDEA. Despite these advantages, many accounting professionals are oblivious to its existence, let alone the 
manner in which it can be systematically administered and interpreted to identify anomalies in accounting numbers.

This paper put forward a consistent and concise approach for accounting professionals to operationalise Benford's Law through IDEA. The methodology was demonstrated on two real-world datasets in the public domain comprising of several thousand instances. It examined transactions complying with Benford's distribution, and transactions that did not fit Benford's expected pattern which signifies possible anomalies. The method described in this paper can be cost effectively implemented by accounting professionals on whole populations of interest rather than resorting to sampling. This can also serve as an effective reasonableness check for auditors in identifying high risk areas that can enable more extensive audit coverage corresponding to the established risk. If properly implemented, Benford's Law can be a valuable aid for accounting professionals in light of the considerable costs associated with economic crime. Future research should explore accounting professionals' perceptions and attitudes towards the practical utility of Benford's Law in identifying suspect transactions. If the extent of implementing Benford's Law in practice is found to be lacking, professional bodies should promote its value given its proven ability to identify anomalies in accounting numbers.

\section{References}

Amouzegar, M., \& Moshirvaziri, K. (2018). Information Security and Benford's Law - Decision Sciences Institute. Retrieved 6 April 2020, from https://decisionsciences.org/information-security-and-benfords-law/

Benford, F. (1938). The Law of Anomalous Numbers. Proceedings of the American Philosophical Society, $78(4), 551-572$.

Bhattacharya, S., Xu, D., \& Kumar, K. (2011). An ANN-based auditor decision support system using Benford's law. Decision Support Systems, 50(3), 576-584. https://doi.org/10.1016/j.dss.2010.08.011

Boyle, J. (1994). An Application of Fourier Series to the Most Significant Digit Problem. The American Mathematical Monthly, 101(9), 879-886. https://doi.org/10.1080/00029890.1994.11997041

Carreira, P., \& Silva, C. G. da. (2016). Assessing the omission of records from a data set using Benford's law. Journal of Financial Crime. https://doi.org/10.1108/JFC-10-2015-0060

Carslaw, C. (1988). Anomalies in Income Numbers: Evidence of Goal Oriented Behaviour. The Accounting Review, 63(2), 321-327.

Cho, W. K. T., \& Gaines, B. J. (2007). Breaking the (Benford) Law. The American Statistician, 61(3), $218-223$. https://doi.org/10.1198/000313007X223496

Collins, C. (2017). Using Excel and Benford's Law to detect fraud. Journal of Accountancy, April. https://www.journalofaccountancy.com/issues/2017/apr/excel-and-benfords-law-to-detect-fraud.html

Durtschi, C., Hillison, W., \& Pacini, C. (2004). The Effective Use of Benford's Law to Assist in Detecting Fraud in Accounting Data. Journal of Forensic Accounting, 5, 17-33.

Gee, J. (2018). The Financial Cost of Fraud 2018: The latest data from around the world. Crowe Audit, 1-20.

Hill, T. (1995). A Statistical Derivation of the Significant-Digit Law. Statistical Science, 10(4), 354-363. https://doi.org/10.1214/ss/1177009869

Hill, T. (1998). The First Digital Phenomenon. American Scientist, 86(4), 358-363. https://doi.org/10.1511/1998.31.815

IDEA. (2018). The Complete Guide to Auditing with Data Analytics Tools. Retrieved 6 April 2020, from IDEA. https://idea.caseware.com/the-complete-guide-to-auditing-with-data-analytics-tools/

IIARF, IIARF. (2009). IT Audit Benchmarking Study: Executive Summary and Report, Altamonte. Institute of Internal Auditors Research Foundation.

Kim, H.J., Mannino, M., \& Nieschwietz, R. (2009). Information technology acceptance in the internal audit profession: Impact of technology features and complexity. International Journal of Accounting Information Systems, 10, 214-228. https://doi.org/10.1016/j.accinf.2009.09.001

Kruger, P., \& Yadavalli, S. (2017). The power of one: Benford's Law. South African Journal of Industrial Engineering, 28. https://doi.org/10.7166/28-2-1753

Kuruppu, N. (2019). The Application of Benford's Law in Fraud Detection: A Systematic Methodology. International Business Research, 12(10), 1-10. https://doi.org/10.5539/ibr.v12n10p1

Kyd, C. (2017). Use Benford's Law with Excel to Improve Business Planning. http://www.exceluser.com/ideas/benford_xl12.htm 
Lavion, D. (2018). Pulling fraud out of the shadows: Global Economic Crime and Fraud Survey 2018. $P w C$ Global Economic Crime and Fraud Survey, 1-30.

Matthews, R. (1999). The power of one. New Scientist. Retrieved from https://www.newscientist.com/article/mg16321944-600-the-power-of-one/

Moolman, A. M. (2017). The usefulness of analytical procedures, other than ratio and trend analysis, for auditor decisions. International Business \& Economics Research Journal (IBER), 16(3), 171. https://doi.org/10.19030/iber.v16i3.9976

Newcomb, S. (1881). Note on the Frequency of Use of the Different Digits in Natural Numbers. American Journal of Mathematics, 4(1), 39-40. https://doi.org/10.2307/2369148

Nigrini, M. J. (1999). I've got your number. Journal of Accountancy, 187(5), 79-83.

Nigrini, M. J. (2018). Round numbers: A fingerprint of fraud. Journal of Accountancy. https://www.journalofaccountancy.com/issues/2018/may/fraud-round-numbers.html

Nigrini, M. J. (2019). The patterns of the numbers used in occupational fraud schemes. Managerial Auditing Journal, 34(5), 606-626. https://doi.org/10.1108/MAJ-11-2017-1717

Nigrini, M. J., \& Mittermaier, L. J. (1997). The Use of Benford's Law as an Aid in Analytical Procedures. Auditing: A Journal of Practice \& Theory, 16(2), 52.

Oregon Audit. (2016). How to: Use Benford's law in ACL. Retrieved 6 April 2020, from Oregon Auditing. https://oregonaudits.org/2016/01/25/how-to-use-benfords-law-in-acl/

Pomykacz, M., Olmsted, C., \& Tantinan, K. (2017). Benford's Law in Appraisal. The Appraisal Journal, Fall, 274-284.

PricewaterhouseCoopers. (2020). Global Economic Crime and Fraud Survey 2020. PwC. Retrieved 6 April 2020, from https://www.pwc.com/gx/en/services/advisory/forensics/economic-crime-survey.html

Silva, W. B., Travassos, S. K. M., \& Costa, J. I. F. (2017). Using the Newcomb-Benford Law as a Deviation Identification Method in Continuous Auditing Environments: A Proposal for Detecting Deviations over Time. Revista Contabilidade \& Finanças, 28(73), 11-26. https://doi.org/10.1590/1808-057x201702690

Simkin, M. (2010). Using Spreadsheets and Benford's Law to Test Accounting Data. ISACA Journal, 1, 1-5.

Singleton, T. W. (2011). Understanding and Applying Benford's Law. ISACA Journal, 3, 4.

Sweet, P. (2018). Global cost of fraud tops $£ 3$ trillion. Retrieved 6 April 2020, from Accountancy Daily. https://www.accountancydaily.co/global-cost-fraud-tops-ps3-trillion

Thomas, J. K. (1989). Unusual Patterns in Reported Earnings. The Accounting Review, 64(4), 773-787.

Watrin, C., Struffert, R., \& Ullmann, R. (2008). Benford's Law: An instrument for selecting tax audit targets? Review of Managerial Science, 2(3), 219. https://doi.org/10.1007/s11846-008-0019-9

Weinberg, R., \& Busta, B. (1998). Using Benford's law and neural networks as a review procedure. Managerial Auditing Journal, 13(6), 356-366. https://doi.org/10.1108/02686909810222375

Wells, J. T. (2013). Corporate Fraud Handbook: Prevention and Detection (4th ed.). Wiley.

Baker, N. (2009). Software Trend Spotting. Retrieved 6 April 2020, fromhttp://www.theiia.org/intAuditor/freefeature/2009/august/software-trend-spotting/

\section{Copyrights}

Copyright for this article is retained by the author(s), with first publication rights granted to the journal.

This is an open-access article distributed under the terms and conditions of the Creative Commons Attribution license (http://creativecommons.org/licenses/by/4.0/). 